\title{
Beat of Frequency Modes with an Artificial Negative Frequency in Spectrogram Analysis
}

\author{
Atsushi Yabushita, ${ }^{* 1}$ Chih-Hsien Kao, ${ }^{1}$ Yu-Hsien Lee, ${ }^{1}$ and Takayoshi Kobayashi ${ }^{1,2,3,4}$ \\ ${ }^{1}$ Department of Electrophysics, National Chiao Tung University, Hsinchu 300, Taiwan \\ ${ }^{2}$ University of Electro-Communications, 1-5-1 Chofugaoka, Chofu, Tokyo 182-8585 \\ ${ }^{3}$ Institute of Laser Engineering, Osaka University, 2-6 Yamada-oka, Suita, Osaka 565-0971 \\ ${ }^{4}$ ICORP, JST, 4-1-8 Honcho, Kawaguchi, Saitama 332-0012
}

(Received September 29, 2010; CL-100834; E-mail: yabushita@mail.nctu.edu.tw)

Spectrograms have been used for time-resolved analysis of Fourier power spectra in various fields. We have performed spectrogram analysis for two neighboring modes and a low frequency mode changing gate width of the time gate function. The obtained spectrogram of the former showed both amplitude and frequency modulations between two neighboring modes. A contamination appearing on the spectrogram of the latter reflects an artifact showing beat between the signal mode and a negative frequency mode whose sign was inverted from the signal mode. Surprising results from the amplitude and phase modulations were observed in the simulation even in the case of single mode. This puzzling result was explained in terms of the interference between the single mode and the "negative" frequency composing the single mode with the corresponding "positive frequency."

Data which contains more than one vibration mode can be studied by frequency analysis. The most common method for frequency analysis is fast Fourier transform (FFT). ${ }^{1}$ However, being based on the assumption that the signal is stationary, FFT is not applicable for nonstationary and/or nonlinear signals. Time-frequency analysis is analysis for a spectrum which varies with time. The time-frequency analysis does not assume stationary condition of the signal; therefore, it can be used to study real-time change of signal frequency. Short time Fourier transform $(\mathrm{STFT})^{2}$ is one of the most popular methods for timefrequency analysis. The trace obtained in the STFT is called a spectrogram, ${ }^{3}$ which has been used for time-resolved analysis of Fourier power spectra in various research fields. In this work, we have performed FFT and spectrogram analysis for time-resolved absorption change observed for a film of $\operatorname{poly}(p$-phenylenevinylene) derivative.

In this work, a 10-fs laser pulse was used to demonstrate pump-probe dynamics in a film of poly[2-methoxy-5-(2'-ethylhexyloxy)- $p$-phenylenevinylene] (MEH-PPV). ${ }^{4}$ Figure 1a shows the time-resolved absorption change trace $(\Delta A)$ measured at the probe wavelength of $616 \mathrm{~nm}$ scanning delay time from -390 to $1396 \mathrm{fs}$ in $3.6 \mathrm{fs}$ steps. The peaks of the Fourier power spectrum of the $\Delta A$ trace in good agreement with the Raman spectrum $^{5}$ were assigned with the help of the latter as follows. The modes around 970 and $1580 \mathrm{~cm}^{-1}$ correspond to $\mathrm{CH}$ out-ofplane bending and vinyl $\mathrm{C}=\mathrm{C}$ stretching, respectively. $\mathrm{CH}$ inplane bending appears as three peaks around 1110, 1290, and $1310 \mathrm{~cm}^{-1}$.

Time-dependent change of vibration frequencies was studied by spectrogram analysis using the Blackman window function. Supporting Information ${ }^{8}$ shows why the Blackman window was used. The spectrogram analysis was performed (a)

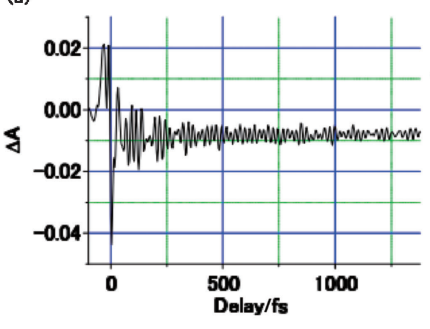

(d)

(b)

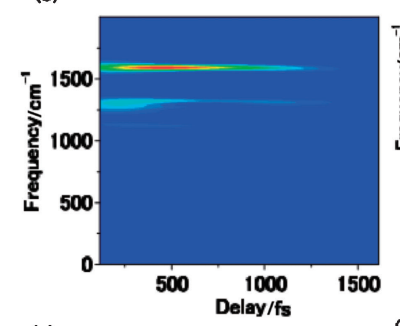

(e)
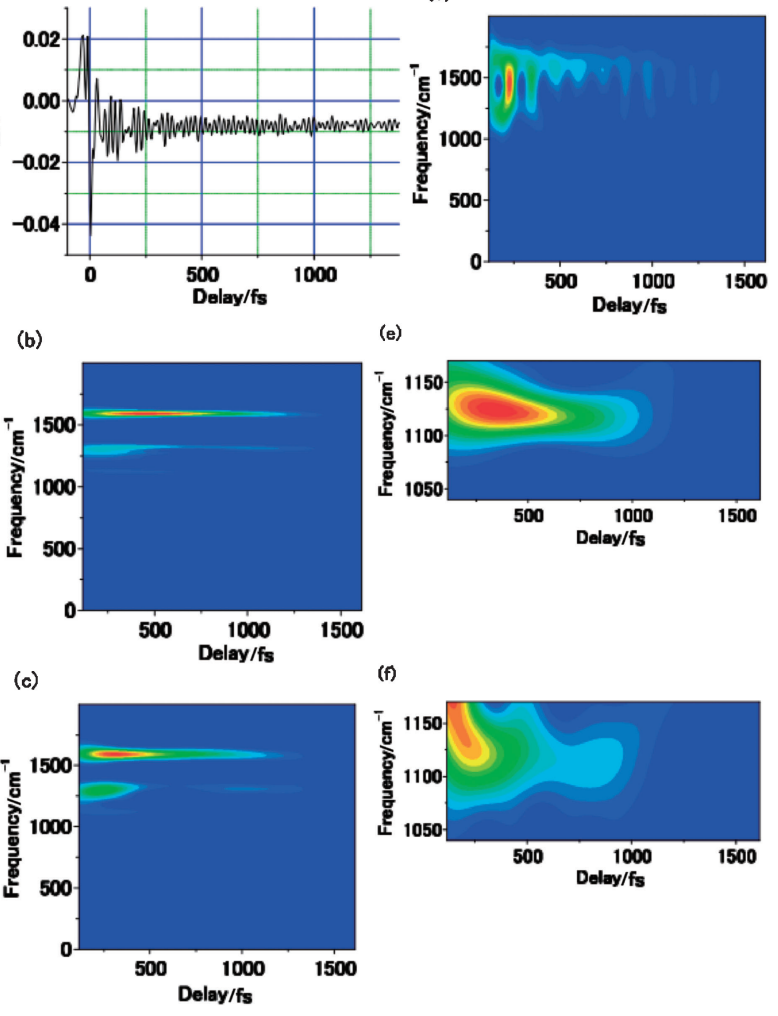

Figure 1. (a) $\Delta A$ trace probed at $616 \mathrm{~nm}$ for delay times of -390 to 1396 fs. (b-f) Spectrograms calculated from the $\Delta A$ trace. The window width used for the calculation was (b) 1600 , (c) 800, and (d) 200 fs. Spectrograms shown in (b) and (c) were clipped from 1040 to $1170 \mathrm{~cm}^{-1}$ and plotted in (e) and (f) respectively.

after removing exponentially decaying signal from the real-time trace. The decay signal was removed by applying the same method as in the FFT analysis mentioned earlier. Figures $1 \mathrm{~b}, 1 \mathrm{c}$, and $1 \mathrm{~d}$ show the spectrograms calculated changing window width of the window function to 1600,800 , and $200 \mathrm{fs}$, respectively. Spectrograms of Figures $1 \mathrm{~b}$ and 1c were clipped between 1040 and $1170 \mathrm{~cm}^{-1}$ to be shown in Figures 1e and 1f, respectively.

Among Figures 1b-1d, Figure $1 \mathrm{~b}$ shows the narrowest frequency width of the mode at $1580 \mathrm{~cm}^{-1}$; however, intensity beat of the mode around $1300 \mathrm{~cm}^{-1}$ is hard to observe. It means that a wider window gives finer frequency resolution and poorer time resolution. The difference of the time resolution is evident by comparison of Figures $1 \mathrm{e}$ and 1f. Figure 1f shows that the 
frequency of the mode around $1110 \mathrm{~cm}^{-1}$ is modulating at a period of ca. $400 \mathrm{fs}$. However, the frequency modulation could not be recognized in Figure 1e.

The intensity beat observed around $1300 \mathrm{~cm}^{-1}$ in Figure 1c has a period of ca. $600 \mathrm{fs}$. It is caused by the beat between the two modes at 1290 and $1310 \mathrm{~cm}^{-1}$ because the two modes have comparable intensity and the frequency difference between the two modes, $20 \mathrm{~cm}^{-1}$, corresponds to the inverse of the beating period of ca. $600 \mathrm{fs}$.

When we use window a width of $200 \mathrm{fs}$, frequency resolution of the spectrogram is ca. $400 \mathrm{~cm}^{-1}$; therefore, the modes at 1580 and ca. $1300 \mathrm{~cm}^{-1}$ are thought to cause a beating signal of ca. $100 \mathrm{fs}$. This is why Figure 1d shows the intensity beat between 1580 and ca. $1300 \mathrm{~cm}^{-1}$. It is, therefore, important to be careful in the analysis of time-dependent vibration modes. If the frequency and/or amplitude modulation is found with modulation frequency corresponding to the difference frequency the modulation intensity must be carefully studied to make sure that the amount of the modulations are smaller than that obtained by simulation using the amplitude of the neighboring mode(s). ${ }^{6}$

Vibration modes exponentially decaying with the corresponding lifetime of each mode can also be analyzed by using linear prediction and singular value decomposition (LP-SVD). ${ }^{7}$ The LP-SVD analysis of the time-resolved trace confirms that the frequency modulation observed around $1300 \mathrm{~cm}^{-1}$ was caused by the beating artifact between the neighboring two modes and that the frequency modulation around $1110 \mathrm{~cm}^{-1}$ was not an artifact but the real frequency modulation of the mode. ${ }^{8}$

We have calculated a spectrogram for the function $f(t)=$ $\sin (2 \pi t / 50) \mathrm{e}^{-t / 500}$, setting window width of the Blackman window function to 200 and $60 \mathrm{fs}$, and the results plotted in Figures $2 \mathrm{a}$ and $2 \mathrm{~b}$, respectively. Figure $2 \mathrm{~b}$ shows clear intensity beats appearing at a period of $25 \mathrm{fs}$, which is double frequency compared with the original oscillating frequency. However, the double frequency beat was difficult to observe in Figure 2a, in which FWHM (full width at half-maximum) of the window is wider than the beat period. It is surprising that the amplitude and phase are modulated in the simulation even in the case of the single mode.

This puzzling result is thought to be reflecting the interference between the single mode and the "negative" frequency composing the single mode with the corresponding "positive" frequency as explained below. The origin of the double frequency beat signal can be explained as follows. Fourier transform of real function oscillating at a certain frequency $\left(\omega_{0}\right)$ gives a spectrum that has a peak at $\omega_{0}$ and another peak at $-\omega_{0}$. In a similar manner, the negative frequency mode $\left(-\omega_{0}\right)$ is also expected to appear on the spectrogram. Beat frequency between the positive frequency and the negative frequency mode is thought to be equal to the frequency difference between the two modes $\left(\left|\omega_{0}-\left(-\omega_{0}\right)\right|=2 \omega_{0}\right)$. This is the origin of the double frequency beat observed in the spectrogram.

Meanwhile, the reason why the beat was hard to observe in Figure 2a can be explained as follows. FWHMs of the windows in the two spectrograms are broader than the period of the double frequency beat $(25 \mathrm{fs})$. In other words, frequency resolutions of the spectrograms are finer than the frequency difference between positive and negative frequency mode. Therefore, the beat between the two modes did not appear in Figure 2a.
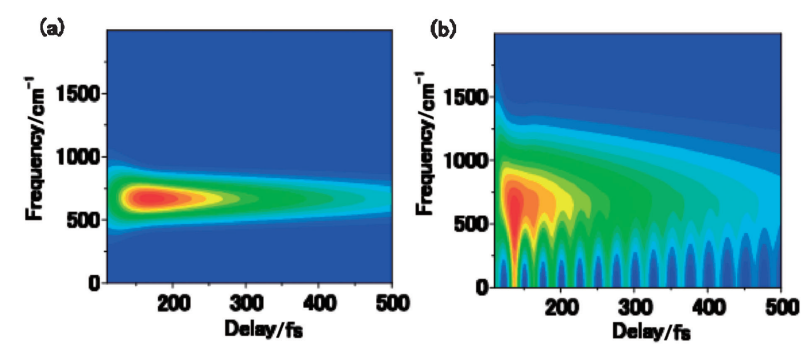

Figure 2. Spectrograms calculated from the function of $\sin (2 \pi t / 50) \exp (-t / 500)$. The window width used for the calculation was (a) 200 and (b) $60 \mathrm{fs}$.

Thus, the fastest time constant studied in the spectrogram analysis limits minimum window width. Meanwhile, maximum window width is limited by the smallest frequency difference between the vibration modes to be observed in the spectrogram analysis. The other restriction for the window width is that FWHM of the window function should be twice as board or more than any oscillation period of the vibration modes in the signal even if the signal contains a single oscillation mode.

This work was supported by a grant to A.Y. from the National Science Council, ROC (NSC 98-2112-M-009-001MY3), and by a grant from the Ministry of Education (MOE) in Taiwan under the ATU Program at National Chiao Tung University to A.Y. and T.K.

\section{References and Notes}

1 M. Heideman, D. Johnson, C. Burrus, IEEE ASSP Mag. 1984, 1, 14; J. W. Cooley, J. W. Tukey, Math. Comput. 1965, 19, 297; J. C. Schatzman, SIAM J. Sci. Comput. 1996, 17, 1150; T. Kobayashi, M. Yasuda, S. Okada, H. Matsuda, H. Nakanishi, Chem. Phys. Lett. 1997, 267, 472; T. Kobayashi, T. Saito, H. Ohtani, Nature 2001, 414, 531.

2 Advanced Topics in Signal Processing, ed. by J. S. Lim, A. V. Oppenheim, Englewood Cliffs, NJ, Prentice-Hall; M. Portnoff, IEEE Trans. Acoust., Speech, Signal Process. 1980, $28,55$.

3 L. Cohen, Proc. IEEE 1989, 77, 941; R. Trebino, D. J. Kane, J. Opt. Soc. Am. A 1993, 10, 1101; S. Rutz, E. Schreiber, Eur. Phys. J. D 1998, 4, 151; T. Meier, S. Mukamel, Phys. Rev. Lett. 1996, 77, 3471; M. J. J. Vrakking, D. M. Villeneuve, A. Stolow, Phys. Rev. A 1996, 54, R37; K. Makino, A. Hirano, K. Shiraki, Y. Maeda, M. Hase, Phys. Rev. B 2009, 80, 245428; M. Hase, M. Kitajima, K. Mizoguchi, S. Nakashima, Physica $B$ 2002, 316-317, 292.

4 M. Liess, S. Jeglinski, Z. V. Vardeny, M. Ozaki, K. Yoshino, Y. Ding, T. Barton, Phys. Rev. B 1997, 56, 15712; S. Abe, J. Phys. Soc. Jpn. 1989, 58, 62; T. Ogawa, T. Takagahara, Phys. Rev. $B$ 1991, 44, 8138.

5 T. P. Nguyen, S. H. Yang, P. Le Rendu, H. Khan, Composites, Part A 2005, 36, 515; K. Honda, Y. Furukawa, H. Nishide, Vib. Spectrosc. 2006, 40, 149.

6 J. Du, T. Kobayashi, Chem. Phys. Lett. 2009, 481, 204.

7 H. Barkhuijsen, R. De Beer, W. M. M. J. Bovee, J. H. N. Creyghton, D. Van Ormondt, Magn. Reson. Med. 1985, 2, 86; H. Barkhuijsen, R. De Beer, D. Van Ormondt, J. Magn. Reson. 1985, 64, 343; A. E. Johnson, A. B. Myers, J. Chem. Phys. 1996, 104, 2497.

8 Supporting Information is available electronically on the CSJ-Journal Web site, http://www.csj.jp/journals/chem-lett/ index.html. 